\title{
Discrepancy between Clinical and Postmortem Diagnosis of Dogs in a Veterinary Medical Teaching Hospital
}

\author{
William Torres Blanca, Lígia Fernandes Gundim, Thaís de Almeida Moreira, \\ Taís Meziara Wilson \& Alessandra Aparecida Medeiros-Ronchi
}

\begin{abstract}
Background: The postmortem examination offers the opportunity to study the processes involved in disease. Although a portion of veterinary medical professionals and students consider the necropsy as a diagnostic tool of purely academic interest, it can provide valuable assistance in formulating health strategies in order to prevent and control animal diseases. The number of necropsies performed in general is higher in universities where the cost is subsidized. In veterinary medicine, studies intended to assess the frequency of necropsy and the discrepancy between clinical and postmortem diagnosis of dogs are rare. The main purpose of the necropsy is to discover the cause of death of dogs, by defining a possible etiology and pathogenesis in order to reach a diagnosis.

Material, Methods \& Results: We used medical records and necropsy records to define the clinical and postmortem diagnosis, respectively. Data relating to deaths was recorded as the number of euthanized dogs and natural deaths in 2014. From the information cause of death, these were categorized as infectious disease, cardiac, gastrointestinal, renal, pulmonary, neurological, metabolic or endocrine disease, neoplastic disease, trauma, or systemic disease. We used the Binomial discrepancy in the comparison of the rates between different years and also to verify the association between discrepancy and the correlation between clinical and postmortem diagnosis of dogs with euthanasia and natural death, with statistical significance $(P<0.05)$. In 2009, 56.81\% $(25 / 44)$ of cases included in the study had a concordance between the clinical and postmortem diagnosis, while $43.19 \%$ (19/44) were discordant. In 2014, it was observed that $71.70 \%$ (76/106) of the diagnosis was confirmed with the necropsy, while $28.30 \%$ (30/106) were discordant. The disagreement rate was higher in $2009(P<0.05)$ and there was a reduction of $14.89 \%$ in the disagreement rate between 2009 and 2014 . Regarding the cause of death, infectious diseases, gastrointestinal disease, and heart disease were the categories in which the discrepancy was higher. It was found that in the group of dogs euthanized, the discrepancy rate was lower compared with the group of dogs that had anatural death $(P<0.05)$.

Discussion: The disagreement rate can be considered high when compared with a veterinary study and similar to those observed in a human study. Decrease in the discrepancy rate in the years, as observed by other authors, that can be attributed to improvements and expansion of diagnostic services of the hospital and better training of veterinarians. The difficulty in determining the etiology of infectious diseases is associated with lack of specific diagnostic tests and the high cost of available tests, which often is not bank rolled by the tutor. Dogs in this study were rarely submitted to diagnostics tests such as electrocardiogram or echocardiogram which explains the high discordance in the diagnosis of heart disease. Distemper is an infectious disease of great importance regarding euthanized animals, especially in cases that progress to central nervous system injuries with extremely poor prognosis and wind up having euthanasia indication. Another common cause of domestic animals euthanasia indication is the occurrence malignant neoplasms, which depends on the progression of the disease and psychological and social conditions of the owner. The results generated herein suggests that infectious, gastrointestinal and cardiac diseases origin tend to have a greater discordance between clinical and postmortem diagnosis, however this rate is decreasing due to improved infrastructure of veterinary centers with better professionals qualification.
\end{abstract}

Keywords: cause of death, euthanasia necropsy, postmortem examination. gmail.com - Tel.: +55 (34) 3225-8432]. Avenida Mato Grosso n. 3289. Campus Umarama, Bloco 2 S. CEP 38405-314 Uberlândia, MG, Brazil. 


\section{INTRODUCTION}

The examination of animals after dead offers the opportunity to study the processes involved in disease. Although various techniques of diagnostic imaging have evolved in recent years, providing adequate information on the morphological changes of organs and tissues, the necropsy also provides firsthand what actually happened over the course of the disease, including tissue changes resulting from processes which may or may not have been detected during the clinical examination [2].

In human medicine, the number of necropsies has declined in recent years $[9,10]$ which has been justified by such factors as lack of or decreased interest of pathologists and clinicians, additional costs, use of molecular diagnostic methods that assist in the clinical diagnosis, and the fear of complaints by family members because of a suspicion of negligence during the medical procedure [9]. Other relevant justification is the incorporation of imaging methods in clinical practice (CT and MRI) that allow for an early and precise diagnosis [11]. The number of necropsies performed in general is higher in universities where the cost is subsidized [3].

In human medicine, studies to assess the rate of discrepancy between the clinical and postmortem diagnosis diagnosis are common. In veterinary medicine, studies intended to assess the frequency of necropsy and the discrepancy between clinical and postmortem diagnosis are rare [3]. One reason for the lack of such studies in veterinary medicine is the difficulty to clearly define, in most cases, the cause of death [4].

The main objective of this study was to determine the rate of discrepancy between the clinical and postmortem diagnosis in dogs and the variation in this rate over time, and to identify which category of cause of death showed a greater discrepancy. A secondary objective was to determine the number of euthanasias and to see if there was an association between euthanasia and the discrepancy rate.

\section{MATERIALS AND METHODS}

\section{Sample}

The study was conducted at the Veterinary Hospital of the Federal University of Uberlândia, in Uberlândia - Minas Gerais. Records in the necropsy report were used to obtain the cause of death data, indexed in the Animal Pathology Laboratory. We collect animal information such as age, sex, and medical record number. Using the medical record number it was possible to analyze and photo scan all medical records to obtain the clinical diagnosis and compare it to the postmortem diagnosis.

\section{Collection and analysis of clinical and pathological diagnosis}

The study included dogs necropsied in 2009 and 2014 who had a clinical and a postmortem diagnosis recorded in its medical records and necropsy form, respectively. Dogs who had an inconclusive diagnosis were excluded from this study.

The postmortem diagnosis was assigned by two pathologists according to the history of the animal, pathological findings and carcass conservation state. In cases where it was necessary to confirm or rule out specific causes of death, samples were taken and subjected to toxicological, histopathological, parasitological and virological/bacterial testing. We assumed as cause of death of dogs that recorded in necropsy form.

The postmortem diagnosis was considered as the correct diagnosis and adopted as a criteria for agreement and disagreement diagnostics. If the postmortem diagnosis did or did not coincide with the clinical diagnosis, this was classified as concordant and discordant, respectively.

The postmortem diagnoses were grouped according to the cause of death and included infectious, cardiac, gastrointestinal, renal, pulmonary, neurological, metabolic or endocrine, neoplastic disease, as well as trauma or systemic disease (such examples being hemorrhagic diathesis, shock, and poisoning).

In assessing the discrepancy rate associated with euthanasia in 2014, the number of euthanized animals and the number of animals that underwent a natural death and were necropsies were obtained. In 2009 , this information was not included in the collection of medical records.

\section{Statistical analysis}

Descriptive statistics were used to calculate the proportion of autopsied dogs and the disagreement rate, and the concordance between the clinical and postmortem diagnosis. We used the Binomial Test to compare the rates between different years and also to verify the correlation between clinical and pathological diagnosis with euthanasia and natural death, with a significance level of $5 \%$. Analyses were conducted using Bioestat 5.0 program [1]. 


\section{RESULTS}

There was an increase of $85.00 \%$ in the number of dogs attended in the veterinary hospital between 2009 and 2014 (Table 1). The number of necropsies performed also increased $56.00 \%$ when comparing the same years. The number of necropsies in relation to the number of animal attended was higher in 2014 $(P=0.0288)$.

In $2009,56.81 \%(25 / 44)$ of cases had a concordance between the clinical and postmortem diagnosis. In 2014 , it was observed that $71.70 \%$ (76/106) of the diagnosis was confirmed with the necropsy, while $28.30 \%$ (30/106) were discordant. The disagreement rate was higher in $2009(P=0.0384)$ and there was a reduction of $14.89 \%$ in the disagreement rate between 2009 and 2014.

When we considered the groups of causes of death in 2009 (Table 1) it was observed that in only two types of cause of death (infectious disease and gastrointestinal disease) the number of disagreements outperformed the number of agreements. For example, there were cases in which the clinical diagnosis was Ehrlichiosis and the disease was peritonitis, or a case with a clinical diagnosis of cardiac dilatation and nephritis, but the necropsy discovered a perforated duodenal ulcer.
When the data were grouped according to the pathological diagnosis in 2014 (Table 1) it was revealed that two categories of cause of death (heart disease and gastrointestinal disease) the number of disagreements exceeded the concordances. Discrepancy occurred, for example, in a case with a clinical diagnosis of leptospirosis and necropsy findings indicating congestive heart failure. Another example case showed a clinical diagnosis of hepatic encephalopathy and the animal had a pancreatic abscess at necropsy.

Regarding euthanasia in 2014, it was observed that $46.22 \%$ (49/106) were euthanized and 10/49 of these euthanized animals showed disagreement and $39 / 49$ cases agreed with the clinical diagnosis. The other $53.78 \%$ (57/106) animals were not euthanized and of these animals 20/57 had a discrepancy and $37 / 57$ showed an agreement with the clinical diagnosis. The disagreement rate washigher when the animal had a natural death than when it was euthanized $(P$ $=0.0472$ ).

In the evaluation of euthanasia according to the cause of death (Table 2)it was revealed that in two categories (infectious disease and neoplastic disease) the number of euthanasias were higher than natural deaths.

Table 1. Agreement and Disagreement diagnoses according to the cause of death of dogs necropsied in 2009 and 2014. Uberlândia-MG, Brazil.

\begin{tabular}{|c|c|c|c|c|c|}
\hline \multirow{2}{*}{ Disease } & \multicolumn{2}{|c|}{2009} & \multicolumn{2}{|c|}{2014} & \multirow[b]{2}{*}{ Total } \\
\hline & Agreement & Discrepancy & Agreement & Discrepancy & \\
\hline Infectious & 2 & 6 & 24 & 5 & 37 \\
\hline Heart & 8 & 1 & 5 & 8 & 22 \\
\hline Gastrointestinal & 2 & 5 & 3 & 6 & 16 \\
\hline Renal & 2 & 1 & 3 & 1 & 7 \\
\hline Neurological & 3 & 2 & 5 & 2 & 12 \\
\hline Pulmonary & 0 & 1 & 1 & 0 & 2 \\
\hline Metabolic/Endocrine & 1 & 1 & 1 & 0 & 3 \\
\hline Neoplasic/ Traumatic & 7 & 2 & 24 & 5 & 38 \\
\hline Systemic & 0 & 0 & 10 & 3 & 13 \\
\hline Total & $25(56.81 \%)$ & $19(43.19 \%)$ & $76(71.70 \%)$ & $30(28.30 \%)$ & 150 \\
\hline
\end{tabular}


Table 2. Number of euthanasia and natural deaths according to the type of death because of necropsied dogs in 2014. Uberlândia-MG, Brazil.

\begin{tabular}{cccc}
\hline Disease & Euthanasia & Natural death & Total \\
\hline Infectious & 18 & 11 & 29 \\
Heart & 5 & 10 & 15 \\
Gastrointestinal & 3 & 4 & 7 \\
Renal & 0 & 4 & 4 \\
Neurological & 2 & 5 & 7 \\
Pulmonary & 0 & 1 & 1 \\
Metabolic/ Endocrine & 0 & 1 & 1 \\
Neoplasic Traumatic/ & 20 & 9 & 29 \\
Sistemic & 1 & 12 & 13 \\
\hline Total & $49(46.22 \%)$ & $57(53.78 \%)$ & $106(100.00 \%)$ \\
\hline
\end{tabular}

\section{DISCUSSION}

In 2009, the disagreement rate can be considered high when compared with a study in the same year in the United States [3], that reported a $14.9 \%$ discrepancy rate. In this study, factors such as a smaller contingent of professionals at the hospital during that year, and the lack of professionals dedicated to different specialties may have contributed to this result.

In a human medicine study [10], that included compiling several studies observed an average discrepancy rate of $23.5 \%$, ranging from 4.1 to $49.8 \%$, which are similar to those observed in our study.

When comparing 2009 with 2014, there was a decrease of $14.89 \%$ in the discrepancy rate, as observed in other study [3] who found a reduction of $22.10 \%$ in the discrepancy rate between 1999 and 2009 and attributed the decreased rate discrepancy to several factors such as incorporation of diagnostic imaging and better training of veterinarians.

In our study, the decrease can be attributed to improvements and expansion of diagnostic services in the clinical laboratory, physical restructuring of the hospital with the purchase of new equipment which better support a diagnosis. There was also the implementation of the medical residency program that allowed an expansion in the number of veterinarians in the veterinary hospital with specialized training in various medical areas of the hospital.
Infectious disease is the most common cause of death in dogs associated with lack of guidance and control of these diseases through vaccination. It is often difficult to determine the etiology of the disease due to the scarcity and cost of diagnostic tests [4]. This reality reported in Italy is similar to what was observed in our study. The costs of diagnostic tests are not always accessible or there is not an interest of the owner to bear these costs to identify the cause of the disease.

Regarding the greater disagreement in the category of heart disease from the data in 2014, ECG and other methods for diagnosis of heart diseases are infrequently used in our study in the veterinary hospital, complicating the diagnosis of heart diseases. In a similar manner, in human patients, only $55 \%$ of critical human patients underwent an electrocardiogram at any stage during their ICU stay [6].

Euthanasia was performed with a greater frequency in the category of infectious diseases, and all 18 cases of euthanasia were carried out in this category with a diagnosis of distemper disease. Canine distemper is an infectious disease that can have acute, subacute or chronic clinical presentations, with cutaneous, gastroenteric, ophthalmic, respiratory and or neurological manifestations. In the case of central nervous system lesions the prognosis is extremely reserved for acute cases with neurological signs, and these cases may result in euthanasia [8]. Moreover, distemper disease was the most diagnosed disease in 
adult dogs in Rio Grande do Sul (Brazil) and the second most prevalent disease in puppies [5].

With regard to neoplasms, it is the third most frequent cause of death in dogs, and euthanasia was carried out in most of these animals [11]. These authors also report that mammary tumors were the primary neoplastic disorder that resulted in the deaths of $40.9 \%$ $(9 / 22)$ of dogs. Euthanasia is frequently elected to stop the progression of malignant neoplasms in affected animals [7]. This can be seen routinely in the clinic, based on the animal's condition and often the social and psychological conditions of the owners.

\section{CONCLUSION}

The discrepancy rate decreased between the years and the categories of cause of death that showed a greater dissent were infectious, gastrointestinal, and heart decrease. The discrepancy rate in the group of animals with a natural death was higher. Therefore the adoption of euthanasia occurred mostly in cases with no possibility of cure based on a clinical and postmortem diagnosis.

Declaration of interest. The authors report no conflicts of interest. The authors alone are responsible for the content and writing of paper.

\section{REFERENCES}

1 Ayres M., Ayres L.M., Ayres D.L.\& Santos A.A. 2007. BIOESTAT - Aplicações estatísticas nas áreas das ciências biomédicas. Belém: IDSM, 364p.

2 Cabana J.E. \& Cooper M.E. 2008. Routine necropsy. In: Veterinary necropsy procedure. Muñoz: CLSU Alumni Association Inc., pp.22-25.

3 Dank G., Segev G., Moshe D. \& Kent M.S. 2012. Follow-up study comparing necropsy rates and discrepancies between clinical and pathologic diagnoses at a veterinary teaching hospital. 2009 versus 1989 and 1999. Journal of Small Animal Practice. 53(12): 679-683.

4 Eleni C., Scholl F. \& Scaramozzino P. 2013. Causes of death in dogs in the province of Rome (Italy). Veterinaria Italiana. 50(2): 137-143.

5 Fighera R.A., Souza T.M., Silva M.C., Brum J.S., Graça D.L. \& Kommers G.D. 2008. Causas de morte e razões para eutanásia de cães da Mesorregião do Centro Ocidental Rio-Grandense. Pesquisa Veterinária Brasileira. 28(4): 223-230.

6 Perkins G.D., McAuley D.F., Davies S. \& Gao F. 2003. Discrepancies between clinical and postmortem diagnoses in critically ill patients: an observational study. Critical Care. 7(6): 129-132.

7 Queiroga F. \& Lopes C. 2012. Tumores mamários caninos, pesquisa de novos fatores de prognóstico. Revista Portuguesa de Ciências Veterinárias. 97(543): 119-120.

8 Sherding R.G. 2008. Cinomosecanina. In: Birchard S.J. \& Sherding R.G. (Eds). Manual Saunders de clínica de pequenos animais. 3.ed. São Paulo: Roca, pp.158-162.

9 Shojania K.G., Burton E.C., Tavora F., Crowdes C.D., Sun C.C. \& Burke A.P. 2008. The vanishing non forensic autopsy. New England Journal of Medicine. 358(9): 873-875.

10 Shojania K.G., Burton E.C., Mcdonald K.M. \& Goldman L. 2003. Changes in rates of autopsy-detected diagnostic errors over time: a systematic review. JAMA. 289(21): 2849-2856.

11 Thurnheer R., Hoes C., Doeneck C., Moll C., Muntywiller J. \& Krause M. 2009. Diagnostic performance in a primary referral hospital assessed by autopsy: evolution over a ten-year period. European Journal of Internal Medicine. 20(8): 784-787.

12 Trapp S.M., Iacuzio A.I., Barca Junior F.A., Kemper B., Silva L.C., Okano W., Tanaka N.M., Grecco F.C.A.R., Cunha Filho L.F.C. \& Sterza F.A.M. 2010. Causas de óbito e razões para eutanásia em uma população hospitalar de cães e gatos. Brazilian Jounal of Veterinary Research and Animal Science. 47(5): 395-402.

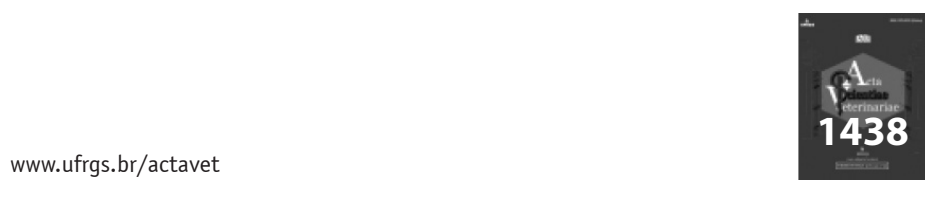

